\title{
Aplicaciones del láser de diodo \\ en Odontología
}

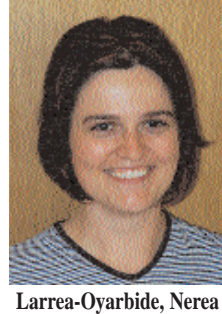

\section{Applications of diode laser in dentistry}

\section{Larrea-Oyarbide, Nerea* \\ España-Tost, Antonio Jesús** \\ B e r i n i - A y t é $s$, Leonardo*** \\ G a y - E s c o d a , Cosme $^{\star \star \star *}$}

*Odontóloga. Residente del Máster de Cirugía e Implantología Bucal. Facultad de Odontología de la Universidad de Barcelona.

**Médico Estomatólogo. Profesor Asociado de Cirugía Bucal. Profesor del Máster de Cirugía Bucal e Impantología Bucofacial. Facultad de Odontología de la Universidad de Barcelona.

***Médico Estomatólogo. Especialista en Cirugía Maxilofacial. Profesor Titular de Patología Quirúrgica Bucal y Maxilofacial. Profesor del Máster de Cirugía Bucal e Implantología Bucofacial. Facultad de Odontología de la Universidad de Barcelona.

****Médico Estomatólogo. Especialista en Cirugía Maxilofacial. Catedrático de Patología Quirúrgica Bucal y Maxilofacial. Director del Máster de Cirugía Bucal e Implantología Bucofacial. Cirujano Maxilofacial del Centro Médico Teknon. Barcelona.

\section{Correspondencia}

Cosme Gay Escoda

Centro Médico Teknon

Cl. Vilana 12

08022 - Barcelona

E-mail: cgay@ub.edu

http://www.gayescoda.com
Resumen: El láser de diodo tiene numerosas aplicaciones en la especialidad de Cirugía Bucal siendo utilizado preferentemente para realizar intervenciones quirúrgicas sobre los tejidos blandos siempre que no impliquen un excesivo sangrado. En Endodoncia, Implantología Bucofacial y Periodoncia se emplea por su importante efecto bactericida. También se utiliza en procedimientos de blanqueamiento dentario. Es importante controlar adecuadamente el tiempo de aplicación y la potencia de trabajo para evitar el sobrecalentamiento de los tejidos vecinos, lo que produciría su necrosis. Siempre que se utilice el láser de diodo se debe efectuar la protección ocular recomendada tanto para el profesional, sus ayudantes y personal auxiliar, como para el paciente.

Palabras clave: Láser de Diodo, Láser en Odontología.

Abstract: The diode laser has numerous applications in the specialty of Oral Surgery, being preferentially used for surgical interventions on soft tissues as long as they do not imply an excessive bleeding. In endodontics, implantology and periodontics it is used because of its important bactericidal effect. It is also used for dental bleaching procedures. It is important to appropriately control the time of application and the working power in order to avoid the overheating of the neighbouring tissues and the subsequent necrosis. Whenever the diode laser is used, all the participating persons - clinician, assistants, auxiliary personnel and patient - have to wear the recommended eye protection.

Key words: Diode laser, Laser in Dentistry.

\begin{tabular}{ccc}
\hline Fecha recepción & Fecha última revisión & Fecha aceptación \\
$25-4-2000$ & $26-2-2001$ & $19-3-2001$ \\
\hline
\end{tabular}

BIBLID [1138-123X (2004)9:5; septiembre-octubre 477-612]

Larrea-Oyarbide N, España-Tost AJ, Berini-Aytés L, Gay-Escoda C. Aplicaciones del láser de diodo en Odontología. RCOE 2004;9(5):529-534. 


\section{Introducción y mico}

\section{característi-}

\section{cas generales}

El láser de diodo está constituido por un medio activo sólido, formado por un semiconductor que frecuentemente usa una combinación de galio, arsenio y otros elementos como el aluminio o el indio para transformar la energía eléctrica en energía luminosa. La longitud de onda de estos láseres para su utilización en Odontología comprende un rango desde 800nm hasta $980 \mathrm{~nm}^{1}$. Pueden emitir en modo continuo o en pulsos $y$, debido a su longitud de onda, la energía es fácilmente transportable a través de fibra óptica. Las fibras ópticas pueden variar entre 200, 300 y $600 \mu m$ según el fabricante. La fibra se pone en contacto con los tejidos blandos para los procedimientos de ablación, incisión y excisión. Estas fibras ópticas deben de ser preparadas antes del primer uso y varias veces intraoperatoriamente para garantizar la eficacia del láser. Este proceso, llamado iniciación, consiste en depositar un fino pigmento oscuro al final de la fibra, produciendo el efecto de «hot tip» (estado en el que se encuentra el tip o fibra óptica del láser preparado para su uso tras la aplicación del pigmento oscuro). El láser de diodo emite en el infrarrojo cercano con una potencia comprendida entre $0,5 \mathrm{~W}$ y $15 W^{2}$.

La ventaja de los láseres de diodo es su pequeño tamaño. Algunas unidades son compactas y fáciles de transportar dentro de la clínica dental en un mínimo tiempo, aunque podemos encontrar equipos de hasta $30 \mathrm{~kg}$ de peso. Estos láseres quirúrgicos son actualmente los de menor coste econó-
En España los equipos de láser de diodo que podemos encontrar en el mercado son muy diferentes y sus características técnicas varían en función del fabricante.

Los equipos disponibles en el mercado nacional son el Opus 5 de 830nm $\pm 10 \mathrm{~nm}$ (Opusdent LTD, Netanya, Israel), Opus 10 de $830 \mathrm{~nm} \pm 10 \mathrm{~nm}$ (Opusdent LTD, Netanya, Israel), Smarty A800-900 de 980nm (Deka Mela, Florencia, Italia), DEX 81015 de 810nm (Imex Dental, Madrid, España), Blankident de 810nm (Deka Mela, Florencia, Italia), SmilePro de 980nm (Biolitec, Jena, Alemania) y el LaserSmile de 810nm (Biolase, San Clemente, EE.UU) (fig 1).

Siguiendo las clasificaciones internacionales en cuanto a las medidas de seguridad, tanto de la Unión Europea (ISO) como de los EE.UU. (ANSI), el láser de diodo es de tipo IV. Debido a que el haz de energía láser puede ser reflejado por instrumentos cercanos a la zona operatoria o incluso por el propio tejido irradiado, hay que tomar ciertas precauciones cuando se utiliza esta tecnología. El principal riesgo que implica la manipulación de radiaciones visibles o infrarrojas con longitudes de onda entre los 400 y los 1400nm es la posibilidad de dañar la retina. Por este motivo deben utilizarse como medida de seguridad siempre gafas protectoras especiales, tanto para el paciente como para el profesional y el personal auxiliar. El contacto con las superficies metálicas (instrumental quirúrgico metálico, espejos, amalgama, etc.) deben evitarse ya que si incide el haz de luz láser sobre ellos se puede producir una reflexión de dicho haz. Para evitar la reflexión se recomienda la utilización de instrumental metálico no pulido o de plástico ${ }^{3}$. Una de las normas internacionales de seguridad que debe de cumplir la tecnología láser, es que cada aparato debe poseer una llave de conexión para que sólo pueda ser utilizado por el personal autorizado. Todas las unidades emisoras llevan una rotulación específica del tipo de láser y la clase de riesgo a la que pertenecen, así como el logo internacional identificativo del tipo de láser de que se trata ${ }^{1}$.

En función de la potencia los láseres de diodo pueden clasificarse en dos grandes grupos: de baja potencia y de alta potencia.

1. Los de baja potencia, también llamados láseres blandos (Low Level Laser Therapy), son de baja energía y emiten en la región del espectro infrarrojo cercano ó del rojo $(632,8,670$ y $830 \mathrm{~nm})$, con una potencia media de 1$100 \mathrm{~mW}$. Sus aplicaciones básicas en ciencias de la salud están basadas en sus efectos de bioestimulación de los tejidos y en su acción analgésica-antiinflamatoria.

2. Los de alta potencia son aquellos láseres con potencias de $1 \mathrm{~W}$ hasta $15 W$ o más y con una longitud de onda comprendida entre $810 \mathrm{~nm}$ y $980 \mathrm{~nm}$. Son láseres relativamente nuevos en su aplicación en el campo de la Medicina y de la Odontología².

En este artículo solamente vamos a tratar del láser de diodo de alta potencia.

\section{Aplicaciones del láser de diodo de alta potencia}



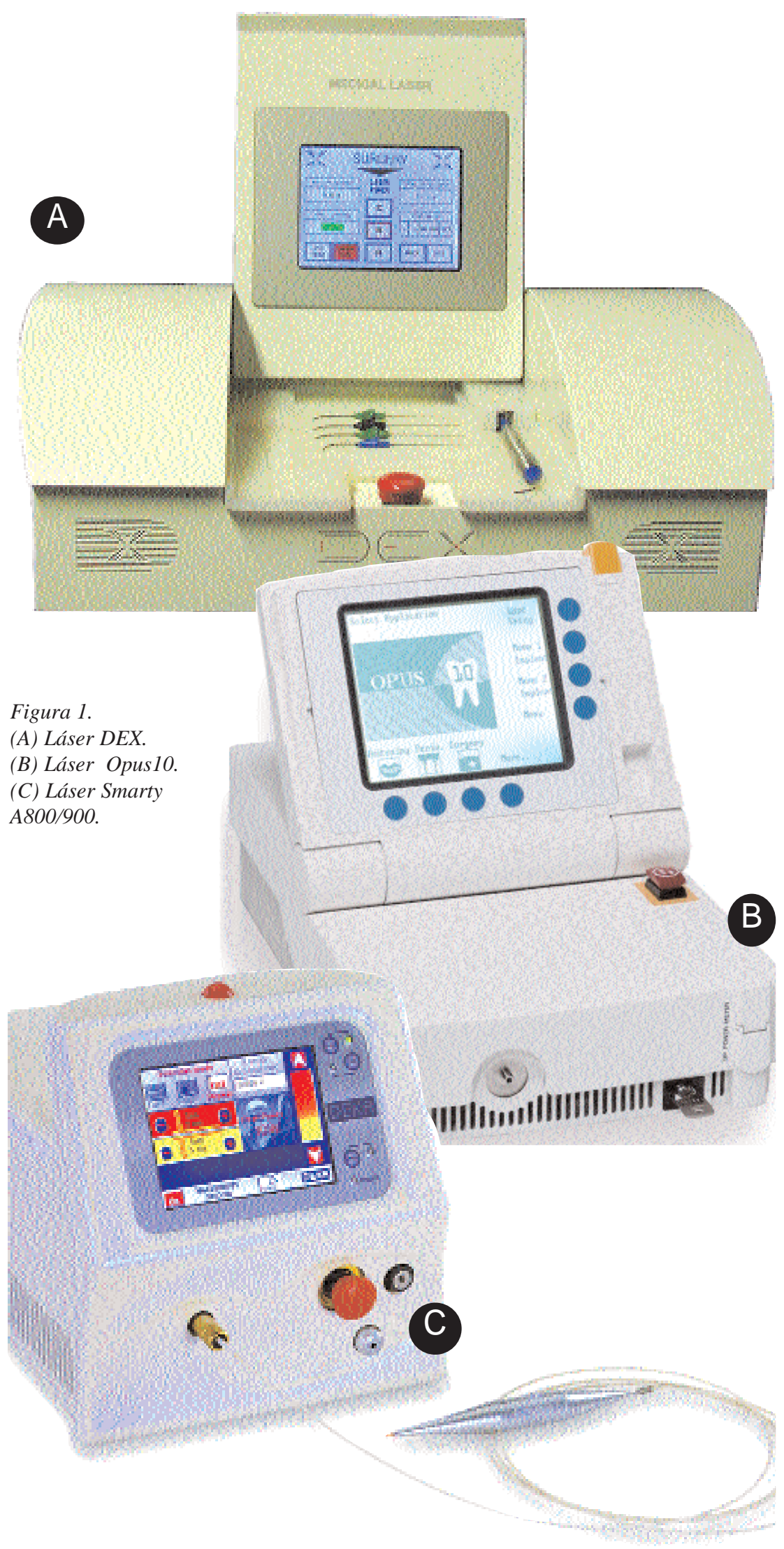

El láser de diodo, debido a su pequeño tamaño y a su bajo coste económico, es actualmente uno de los láseres con más predicamento en Odontología. Las aplicaciones de este tipo de láser se relacionan con su efecto bactericida, con indicaciones específicas en Periodoncia y Endodoncia. También se utiliza en la cirugía de tejidos blandos y para el blanqueamiento dental.

\section{Endodoncia}

Uno de los principales objetivos del tratamiento endodóncico es dejar el conducto radicular libre de microorganismos. Para ello se pueden utilizar diferentes sistemas de desinfección y el láser de diodo es uno de ellos. No obstante se debe de tener precaución con su uso a fin de prevenir el posible daño térmico generado por el láser sobre el periodonto o el propio diente ${ }^{4}$.

Moritz y cols hicieron un estudio in vitro en conductos radiculares con un láser de diodo, con una longitud de onda de 810nm y trabajando a 2, 3 y $4 \mathrm{~W}$ de potencia durante 50 segundos, $\mathrm{y}$ consiguieron una completa eliminación bacteriana cuando empleaban las potencias de 2 y $3 \mathrm{~W}^{5 * *}$. Por otro lado Kreisler y cols compararon la acción del hipoclorito sódico al 0,9\% frente al hipoclorito sódico combinado con un láser de diodo con el fin de conseguir la desinfección de los conductos radiculares. Emplearon una longitud de onda de $809 \mathrm{~nm}$ y potencias de $1 \mathrm{~W}, 3 \mathrm{~W}$ y $4,5 \mathrm{~W}$ durante 60 segundos. Obtuvieron una reducción bacteriana del 99,86\% utilizando conjuntamente el hipoclorito sódico y el láser a $3 \mathrm{~W}$ de potencia. $\mathrm{A}$ pesar de estos datos, su conclusión es que el láser de diodo no puede sustituir al hipoclorito sódico, pero ayuda a obte- 

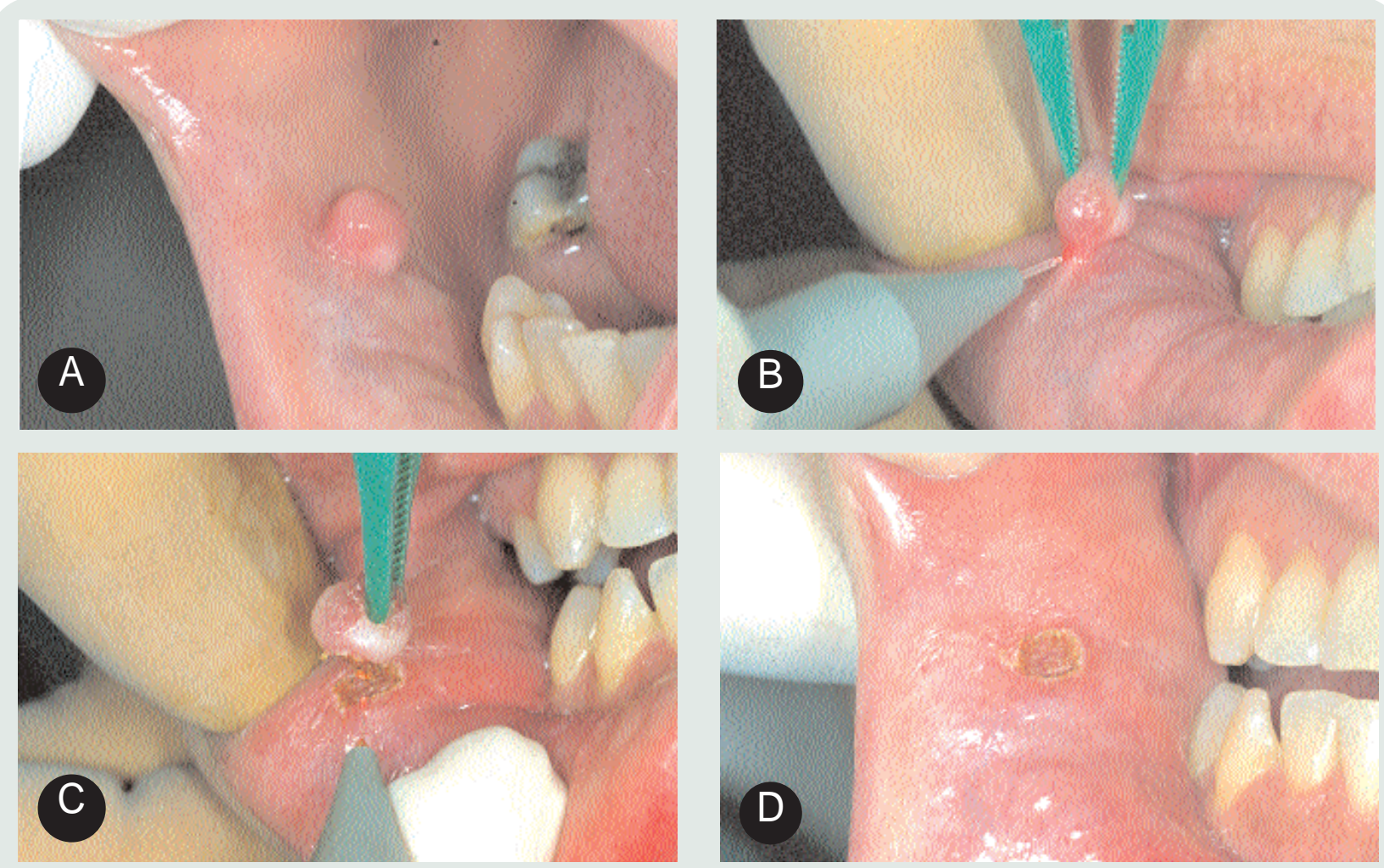

Figura 2. (A) Lesión fibromatosa en la mucosa yugal derecha. (B) Aplicación de la punta de la fibra del láser de Diodo. (C) Exéresis de la lesión. (D) Aspecto de la zona operatoria al finalizar la intervención quirúrgica.

ner mejores resultados ${ }^{6}$

\section{Periodoncia}

En los tratamientos periodontales se pretende obtener la eliminación completa de todos los depósitos calcificados y bacterianos que están situados en las superficies radiculares ${ }^{7}$. La aplicación de la tecnología láser para el control de las enfermedades periodontales está progresando rápidamente en los últimos años. Concretamente el láser de diodo es relativamente nuevo en el tratamiento periodontal. Los procedimientos que se pueden hacer con él son el curetaje de los tejidos blandos, el desbridamiento de las bolsas perio- dontales y todas las incisiones y excisiones gingivales. También tiene gran utilidad en la descontaminación de las bolsas periodontales, ya que consigue eliminar las bacterias anaerobias que contienen ${ }^{8,9}$.

Moritz y cols efectuaron un estudio, en el cual aplicaban un láser de diodo de $805 \mathrm{~nm}$ de longitud de onda a una potencia de $2,5 \mathrm{~W}$, sobre las bolsas periodontales durante 3-4 segundos. Obtuvieron una reducción de la presencia de Actinobacillus actinomycetemcomitans de un $73,5 \%$ y de la Prevotella intermedia de un $85,3 \%{ }^{10}$.

Por otro lado Kreisler y cols utilizaron una longitud de onda de 810nm y una potencia de trabajo entre 0,5 y 2,5W, demostrando que potencias superiores a $1 \mathrm{~W}$ producen carbonización parcial 0 total de la superficie radicular. Observaron además que se obtiene un adecuado efecto antibacteriano empleando este láser a $1 \mathrm{~W}$ de potencia ${ }^{11,12^{* *}}$.

\section{Cirugía bucal}

El láser de diodo es absorbido en menor medida por el agua, y se absorbe más en profundidad que los láseres de Er: YAG, Er:Cr:YSGG y de CO2. Esto permite que la cirugía de los tejidos blandos se pueda realizar en la proximidad de los dientes. El uso del láser quirúrgico de diodo sobre el hueso no 

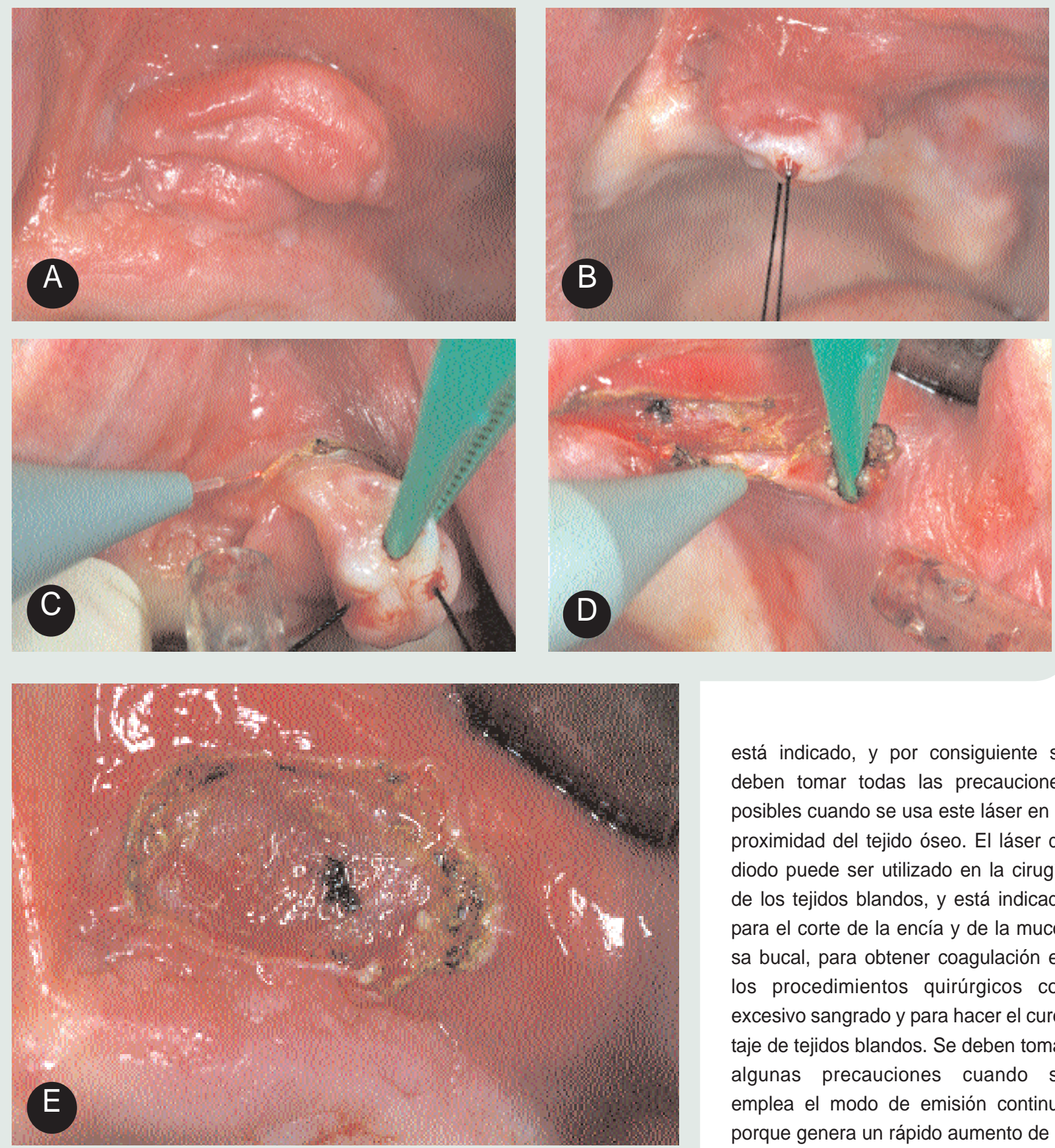

Figura 3. (A) Épulis fisurado a nivel del fondo del vestíbulo. (B) Colocamos un punto de sutura para traccionar la lesión. (C) Exéresis del épulis. (D) Hemostasia para evitar el sangrado intraoperatorio. (E) Postoperatorio inmediato tras la exéresis de la lesión.

está indicado, y por consiguiente se deben tomar todas las precauciones posibles cuando se usa este láser en la proximidad del tejido óseo. El láser de diodo puede ser utilizado en la cirugía de los tejidos blandos, y está indicado para el corte de la encía y de la mucosa bucal, para obtener coagulación en los procedimientos quirúrgicos con excesivo sangrado y para hacer el curetaje de tejidos blandos. Se deben tomar algunas precauciones cuando se emplea el modo de emisión continuo porque genera un rápido aumento de la temperatura en los tejidos sobre los que se trabaja. Las intervenciones quirúrgicas que se pueden hacer con el láser de diodo son: gingivectomía, exéresisbiopsia de tejidos blandos, frenec- 
tomías y vestibuloplastias, etc. ${ }^{13}$ (figs. 2 y 3). Se puede conseguir una adecuada he-mostasia en las lesiones superficiales de la mucosa bucal al desfocalizar el láser, aunque la hemostasia no es tan rápida como la que se consigue con el láser de Argón. Su efecto hemostático es mucho menor en comparación con el láser de $\mathrm{CO}_{2}$, por lo que solo nos será útil para realizar la exéresis de lesiones superficiales y no será recomendable para efectuar la exéresis de lesiones angiomatosas ni para hacer intervenciones quirúrgicas en las que se prevé que se producirá un sangrado profuso ${ }^{14}$.

\section{Implantología}

El hecho de que se haya postulado un efecto inhibitorio del láser de diodo sobre el crecimiento de las bacterias presentes en las bolsas periodontales, fundamenta su indicación en el tratamiento de la periimplantitis. En este sentido, los estudios in vitro realizados por Romanos y cols ${ }^{14^{*}}$ y Hass y cols ${ }^{15}$, en los que el láser de diodo fue aplicado sobre las superficies dentaria e implantaria con una longitud de onda de $905 \mathrm{~nm}$ durante un minuto, parecen conseguir aquel objetivo. Por otro lado, a pesar de que el contacto del haz de luz del láser con el implante provoca un ligero aumento de la temperatura del mismo, el $80-90 \%$ de la radiación es reflejada por su superficie, por lo que no está demostrado que el posible fracaso del tratamiento implantológico pueda ser debido a la absorción de la energía térmica emitida por este láser $^{14-16}$.

\section{Blanqueamiento}

El láser de diodo ha sido ampliamente comercializado para los procedimientos de blanqueamiento dental. La luz del láser de diodo activa el gel del blanqueamiento dental $\left(\mathrm{H}_{2} \mathrm{O}_{2}\right)$ produciendo su descomposición química y acelerando su penetración en el esmalte. Sin embargo, la utilización del láser de diodo en este campo no reduce la aparición de sensibilidad dentinaria. La realidad es que no se obtienen mejores resultados estéticos que con la técnica tradicional, pero tiene la ventaja de que disminuye el tiempo de tratamiento y por tanto es más cómodo para los pacientes ${ }^{15}$.

\section{Bibliografía recomendada}

Para profundizar en la lectura de este tema, el/los autor/es considera/an interesantes los artículos que aparecen señalados del siguiente modo: *de interés **de especial interés.

1. España AJ, Velasco V, Gay Escoda C, Berini L, Arnabat J. Aplicaciones del láser de $\mathrm{CO}_{2}$ en Odontología. Madrid: Ergon,1995.

2. Romanos GE, Everts H, Nentwig GH. Effects of diode and Nd:YAG laser irradiation on titanium discs: A scanning electron microscope examination. J Periodontol 2000;71:810-5.

3. Trullols C, España AJ, Berini L, Gay Escoda C Aplicaciones del láser blando en Odontología. Anal Odontoestomatol 1997;2:45-51.

4. Matsumoto K. Lasers in endodontics. Dent Clin North Am 2000;4:889-905.

5**. Moritz A, Gutknecht N, Goharkhay K, Schoop $\mathrm{U}$, Wernisch J, Sperr W. In vitro irradiation of infected root canals with a diode laser: Results of microbiologic, infrared spectrometric, and stain penetration examinations. Quintessence Int 1997;28:205-9.

En este artículo se presenta un estudio in vitro sobre la aplicación del láser de diodo con una longitud de onda de $810 \mathrm{~nm}$ y a diferentes potencias de 2, 3 y $4 \mathrm{~W}$ durante 50 segundos, con el fin de valorar su efecto antibacteriano, obteniéndose una completa eliminación de los microorganismos al emplear potencias de 2 y $3 \mathrm{~W}$.

6. Kreisler M, Kohnen W, Beck M y cols. Efficacy of $\mathrm{NaOCl} / \mathrm{H}_{2} \mathrm{O}_{2}$ irrigation and $\mathrm{GaAlAs}$ laser in decontamination of root canals in vitro. Lasers Surg Med 2003;32:189-96.

7. Schwarz F, Sculean A, Berakdar M, Szathmari L, Georg T, Becker J. In vivo and in vitro effects of an Er:YAG laser, a GaAlAs diode laser, and scaling and root planing on periodontally diseased root surfaces: A comparative histologic study. Lasers Surg Med 2003; 32:359-66.

8. Herbert I. Use of lasers in periodontics. Dent Clin North Am 2000;4:779-89.

9. Moritz A, Schoop U, Goharkhay K y cols. Treatment of periodontal pockets with a diode laser. Lasers Surg Med 1998;22:302-11.

10. Moritz A, Gutknecht N, Doertbudak O y cols. Bacterial reduction in periodontal pockets through irradiation with a diode laser: A pilot study. J Clin Laser Med Surg 1997;15:33-7.

11. Kreisler M, Meyer C, Stender E, Daublander M, Willershausen-Zonnchen B, D'Hoedt B. Effect of diode laser irradiation on the attachment rate of periodontal ligament cells: An in vitro study. J Periodontol 2001;72:1312-7.

$12 * *$. Kreisler M, Al Haj H, Daublander M y cols. Effect of diode laser irradiation on root surfaces in vitro. J Clin Laser Med Surg 2002; 20:63-9.
En este artículo los autores demuestran que con la utilización del láser de diodo con potencias inferiores a $1 \mathrm{~W}$ de potencia se obtiene un buen efecto antibacteriano en las bolsas periodontales.

13. Strauss R. Lasers in oral and maxillofacial surgery. Dent Clin North Am 2000;4: 851-71.

14*. Romanos G, Nentwig GH. Diode laser (980 $\mathrm{nm}$ ) in oral and maxillofacial surgical procedures: Clinical observations based on clinical applications. J Clin Laser Med Surg 1999; 17:193-7.

Este trabajo propone la utilización del láser de diodo para la cirugía de los tejidos blandos bucofaciales. Se pueden realizar intervenciones quirúrgicas como gingivectomía, biopsias, frenectomías y vestibuloplastias. La capacidad de coagulación de este láser no es tan eficaz como la del láser de $\mathrm{CO} 2$, y por lo tanto no está indicado para la exéresis de lesiones angiomatosas.

15. Haas R, Dortbudak O, Mensdorff-Pouilly N, Mailath G. Elimination of bacteria on different implant surfaces through photosensitization and soft laser: An in vitro study. Clin Oral Implants Res 1997;8:249-54.

16. Sulewski J. Historial survey of lasers dentistry. Dent Clin North Am 2000;4:717-29. 